\title{
Post-concussion syndrome: Correlation of neuropsychological deficits, structural lesions on magnetic resonance imaging and symptoms
}

\author{
S. G. S. Datta, S. V. Pillai, S. L. Rao' , J. M. E. Kovoor², B. A. Chandramouli \\ Departments of Neurosurgery, ${ }^{1}$ Clinical Psychology, ${ }^{2}$ Neuroimaging and Interventional Radiology, National Institute of Mental \\ Health and Neurosciences, Bangalore, India
}

\author{
Address for correspondence: \\ Dr. Shibu V. Pillai, \\ Department of Neurosurgery, \\ NIMHANS, Bangalore, India. \\ E-mail: drshibupillai@hotmail.com
}

PMID: 19934558

DOI: $10.4103 / 0028-3886.57810$

\begin{abstract}
Background: Post-concussion syndrome (PCS) associated with mild traumatic brain injury (MTBI) can cause long-lasting disabilities. Magnetic resonance imaging (MRI) evaluation in these patients may demonstrate structural lesions that correlate with functional deficits on neuropsychological testing. However, little is known about the significance of the relationship between structureal lesions on MRI, functional deficits on neuropsychological evaluation and outcome in patients with MTBI. Aims: To assess neuropsychological deficits and structural lesions on MRI in patients with PCS following MTBI, and to find correlation between these findings and PCS. Settings and Design: Prospective, observational, cohort study in a tertiary hospital. Materials and Methods: The study cohort included consecutive patients with MTBI (three months or more duration) and PCS. All the patients in the cohort had neuropsychological testing using the National Institute of Mental Health and Neurological Sciences Neuropsychological Battery for head injury and also MRI using TI, T2 and FLAIR sequences. Statistical analysis was done using Fisher's Exact test of significance. Results: All the 20 patients evaluated had neuropsychological deficits. Eleven patients had lesions on MRI. Disturbances of sleep, behavior and memory and abnormalties in tests for mental speed were more frequent in patients with lesions on MRI, but were not statically significant $(P=0.08)$. Both the test modalities localized lesions predominantly to the frontal and temporal lobes. All the symptoms observed in the patients were associated with prefrontal dysfunction on neuropsychological testing. Conclusions: Prefrontal dysfunction is invariably associated with PCS following MTBI. Structural lesions on MRI may not always be present but when present may influence the degree or severity of the symptoms.
\end{abstract}

Key words: Minimal traumatic brain injury, post-concussion syndrome, magnetic resonance imaging, neuropsychological deficits

\section{Introduction}

The frequency of mild traumatic brain injury (MTBI) is relatively high but it is often unrecognized and misdiagnosed. ${ }^{[1]}$ Although majority of the affected individuals experience transient symptoms, a significant minority will have persistent and sometimes disabling symptoms, preventing them from returning to premorbid lifestyle. ${ }^{[2-4]}$ Post-concussion syndrome (PCS) characterized by multiple cognitive, behavioral, and somatic symptoms is common following MTBI and is often under-diagnosed or misdiagnosed. Neuropsychological testing in patients with PCS assesses the deficits more precisely and also helps to some extent 
in treatment planning and prognostication. ${ }^{[4]}$ The place of magnetic resonance imaging (MRI) in the diagnostic evaluation of PCS is not clear The relationship between the structural lesions on MRI and the deficits on neuropsychological testing has not been well studied. The lesions commonly seen on MRI are the frontal and temporal lesions seen following head injury. ${ }^{[5]}$ Studies show some correlation between these MRI lesions and the deficits on neuropsychological tests. ${ }^{[6]}$ This study assesses neuropsychological deficits and structural lesions on MRI in patients with PCS following MTBI, and tries to find correlation between these findings and PCS.

\section{Materials and Methods}

This is a prospective, observational, cohort study done in a tertiary hospital. The study cohort included consecutive patients with PCS following MTBI. Informed consent was obtained from all the patients recruited in the study. Inclusion criteria included: (1) History of MTBI, defined as an injury which produced a period of unconsciousness for 30 minutes or less and/or brief retrograde amnesia, a Glasgow Coma Scale score of 14 to 15, no focal neurological deficit, and no intracranial complications; (2) Symptoms suggestive of PCS persistent for three or more months following injury, (3) Glasgow Outcome Score (GOS) of five. ${ }^{[7]}$ Patients were excluded if they had undergone any neurosurgical procedure, or required hospital admission or ICU care after head injury. Patients with neurological, neurosurgical or psychiatric illness were also excluded. The functional brain deficits were assessed by neuropsychological testing, using the National Institute of Mental Health and Neurological Sciences (NIMHANS) Neuropsychological Battery for head injury. ${ }^{[8]}$ All the tests were administered and evaluated by the same two persons. The tests were standardized for the Indian population. The test battery included: Digit symbol substitution (mental speed), digit vigilance test (sustained attention), finger tapping test (motor speed), complex Figure test (visual-spatial construction, visual learning and memory), auditory verbal learning test (verbal learning and memory), Verbal N Back test (working memory), Wisconsin card sorting test (set shifting ability), animal names' test (category fluency), Stroop color test (response inhibition), Token test (comprehension ability), and Tower of London test (planning ability). The cutoff scores and percentiles were obtained matched by age, sex, and literacy from the normative data in the NIMHANS Neuropsychology Battery-2004-Manual. ${ }^{[8]}$ A score below the fifteenth percentile was considered deficient. MRI (T1, T2 and FLAIR sequences) was done at the same time as the neuropsychological testing to detect structural lesions. The images were obtained using a 1.5 Tesla Superconducting MRI unit (Siemens Magnetom Vision, Ehrlangen, Germany) and were analyzed by a single radiologist who was blinded to the patient data. Statistical analysis was done using SPSS 10 protocol and Fisher's Exact test of significance was applied.

\section{Results}

The study cohort included 20 patients (males 15, mean age 35 years, median age 35 years, range 23-70 years,). The Glasgow Coma Scale score at the time of traumatic brain injury was 14 or 15 . The mean duration of PCS following MTBI was 28 months (range 3-84, Median 13.5). The mechanism of head injury was road traffic accident (RTA) in 12 patients, falls in four, assault in one, and other injuries in three. Neurological symptoms in the patients are given in Table 1. Fourteen patients had school education, three college education, and three were illiterate. Deficits on neuropsychological tests were detected in all the patients [Figure 1]. The most frequently seen deficits on neuropsychological tests included: Verbal learning deficits (85\%), verbal memory deficits (75\%), difficulties in sustaining attention (55\%), impaired planning (55\%), and chages in response inhibition (55\%).

The possible anatomical substrate for the deficits on neuropsychological tests is shown in Figure 2. The most common anatomical substrate for the neuropsychological deficits was bilateral prefrontal lobes. MRI was normal in nine patients (Group A) and abnormal in 11 patients

\begin{tabular}{lc}
\hline Table 1: Presenting complaints of the patients & \\
\hline Symptoms & Number (\%) \\
\hline Headache & $16(80)$ \\
Visual disturbance & $5(25)$ \\
Sleep disturbances & $14(70)$ \\
Behavioral/emotional/mood disturbances & $14(70)$ \\
Memory deficits & $15(75)$ \\
Attention/judgmental deficits & $11(55)$ \\
Motivational problems & $7(35)$ \\
Giddiness & $9(45)$ \\
\hline
\end{tabular}

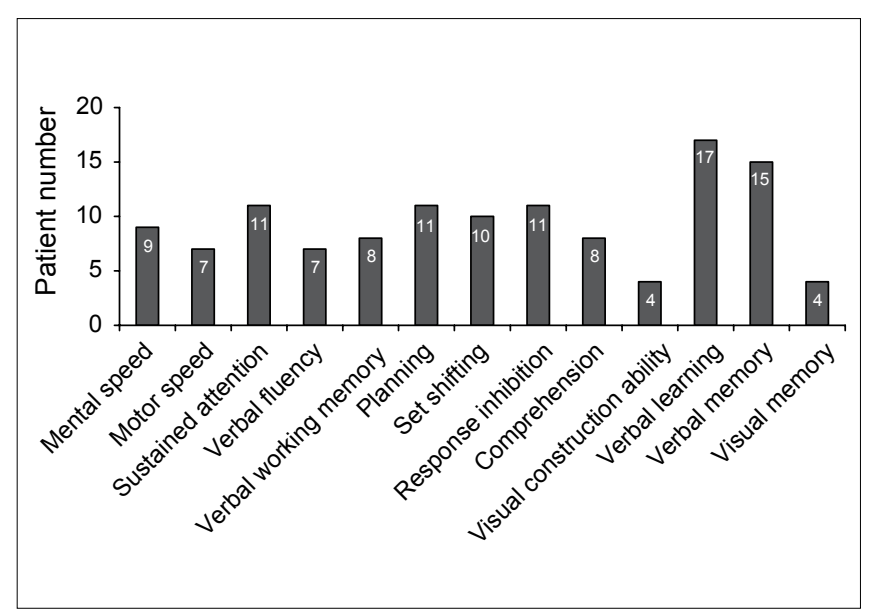

Figure 1: Number of patients with impaired neuropsychological test results 
(Group B) [Table 2]. In patients with MRI lesions, the lesions were located in frontal lobes in 11 and seven patients had in addition lesions in temporal lobes, one patient had lesion in the occipital lobe, and one patient had lesion in deep callosal lesion. Combined cortical and sub-cortical lesions were seen in six patients, sub-cortical alone in five patients, and callosal in one patient. On $\mathrm{T} 1$ images the lesions were hypointense in eight cases, and isointense in three cases. On T2 weighted images the lesions were hypointense in one and hyperintense in 10 cases. There was no correlation between lesion intensity on MRI and neuropsychological deficits.

In the 11 patients with MRI findings, the mechanism of injury was RTA in nine patients, one patient had an industrial accident and the last was an assault. The symptom profile of patients in Group A and B is shown in Figure 3. Patients with lesions on MRI were more likely to have disturbances of sleep, behavior and memory. However, these differences were not statistically significant.

On comparing the neuropsychological test results of patients in Groups A and B, the only notable difference found was in tests for mental speed $[P=0.08$, Figure 4]. In patients with PCS there was no significant correlation between any of the symptoms and deficits on neuropsychological tests [Figure 5]. Most of the symptoms were associated with prefrontal dysfunction. Frontal

Table 2: Magnetic resonance imaging lesions' characteristics seen in 11 patients

\begin{tabular}{lcc}
\hline Location & Frontal (11) & Temporal (7) \\
\hline Right side & 8 & 2 \\
Left side & 3 & 4 \\
Bilateral & 0 & 1 \\
Volume $\left(\mathrm{mm}^{3}\right)$ & $1-17$ (mean 6.5) & $4-78$ (mean 28.76) \\
Grey matter & 0 & 1 \\
White matter & 5 & 1 \\
Grey and white & 6 & 5 \\
\hline
\end{tabular}

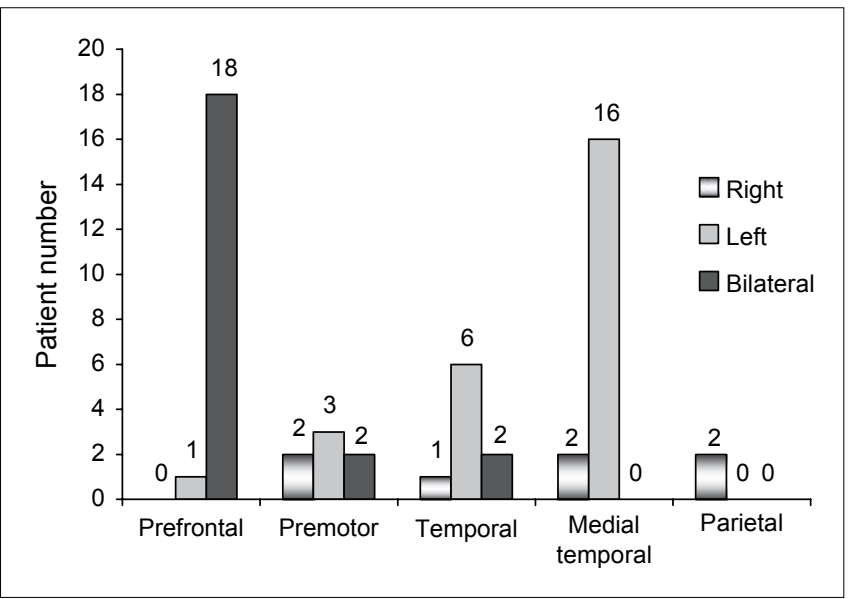

Figure 2: Neuropsychological localization of deficits in all patients tested lesions on MRI had a sensitivity of $55 \%$ (11/20 patients) in detecting frontal lobe deficits on neuropsychological tests [Table3]. Also MRI had a sensitivity of 29\% (5/17 patients) and specificity of $33 \%$ (1/3 patients) in detecting temporal lobe deficits on neuropsychological tests [Table 4].

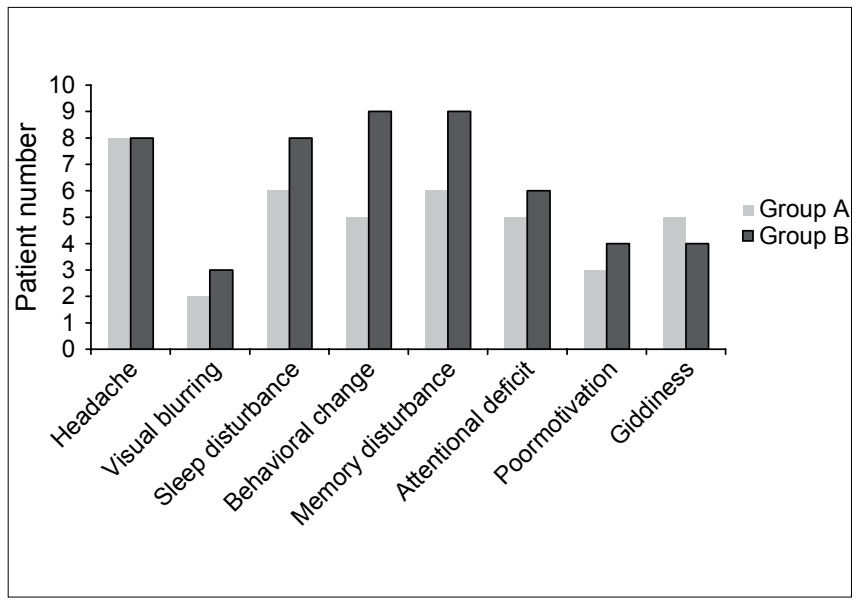

Figure 3: Symptom profile of patients without (group A) and with (group B) lesions on magnetic resonace imaging

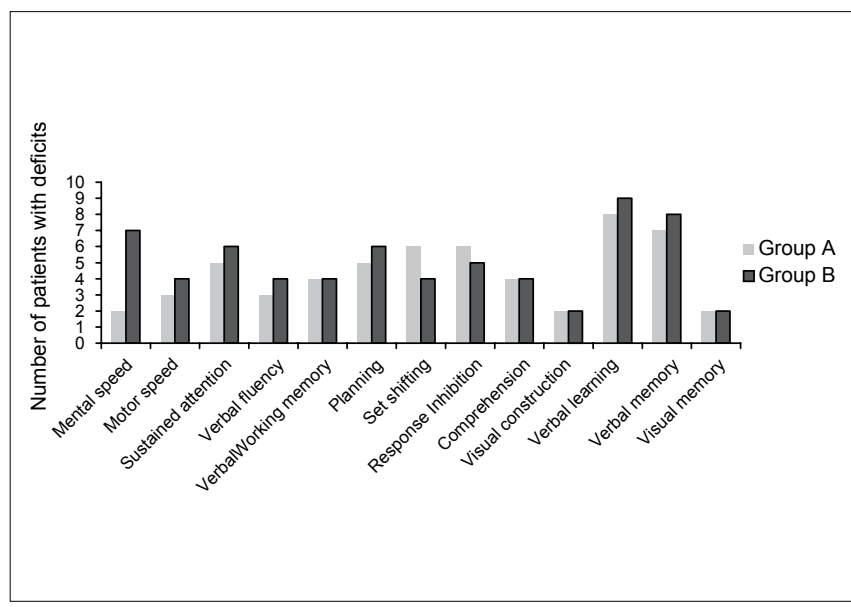

Figure 4: Neuropsychological test result of patients without (group A) and with (group B) lesions on magnetic resonace imaging

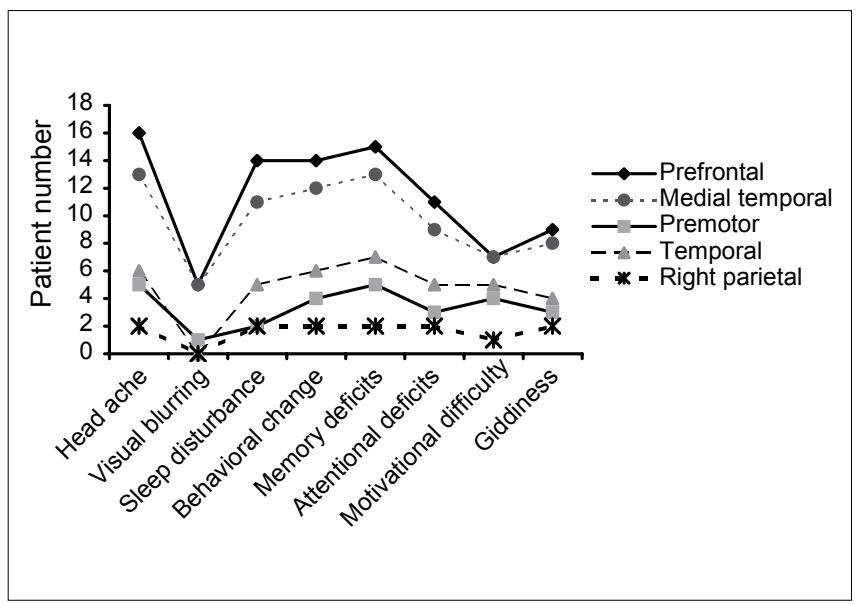

Figure 5: Comparison of PCS symptoms with localisation of neuropsychological deficits 


\begin{tabular}{l|ccc}
\hline $\begin{array}{l}\text { Table 3: Neuropsychological deficit localization in patients } \\
\text { with frontal lesions on magnetic resonance imaging }\end{array}$ \\
\hline Frontal MRI lesions & $\begin{array}{l}\text { Frontal neuropsychological deficit } \\
\text { localization }\end{array}$ \\
\cline { 2 - 4 } & Present & Absent & Total \\
\hline Present & 11 & 0 & 11 \\
Absent & 9 & 0 & 9 \\
Total & 20 & 0 & 20 \\
\hline
\end{tabular}

\begin{tabular}{l|ccc}
\hline $\begin{array}{l}\text { Table 4: Neuropsychological deficit localization in patients } \\
\text { with temporal lesions on magnetic resonance imaging }\end{array}$ \\
\hline Temporal MRI lesions & \multicolumn{4}{c}{ Temporal neuropsychological deficit } \\
& localization \\
\cline { 2 - 4 } & Present & Absent & Total \\
\hline Present & 5 & 2 & 7 \\
Absent & 12 & 1 & 13 \\
Total & 17 & 3 & 20 \\
\hline
\end{tabular}

In patients with exclusive frontal lesion on MRI $(\mathrm{N}=4)$, localization on neuropsychological tests was to the bilateral prefrontal lobe in all the patients. In addition, two patients had right premotor dysfunction, one patient had left premotor dysfunction, one had left temporal dysfunction and four had left medial temporal dysfunction. In patients with frontal and temporal lesions on MRI ( $\mathrm{N}=7)$, localization on neuropsychological tests was to the bilateral prefrontal lobe in six patients. In addition, one patient had left prefrontal dysfunction, one had left premotor dysfunction, two patients had left temporal dysfunction, one had right temporal dysfunction and four had left medial temporal dysfunction.

\section{Discussion}

In this prospective observational study all the twenty patients with PCS following MTBI had deficits on neuropsychological tests and eleven patients had lesions on MRI. Both the test modalities localized the lesions predominantly to the frontal and temporal lobes. All symptoms were associated with prefrontal dysfunction on neuropsychological testing. Structural lesions as detected on MRI may influence the degree or severity of the problem.

The mean age of 35 years reflects the relatively young and productive age profile of the patients. The mean duration since injury was 28 months (range: 3-84 months). These observations suggest that PCS can manifest early and may persist for a long period. Earlier studies have assessed neuropsychological deficits at three months or at one year. ${ }^{[9,10]}$ In this cohort the distribution of neuropsychological deficits was uniform across various time periods. The commonest symptoms reported by patients in this study were headache, memory and sleep disturbances, and behavioral changes similar to the observations in other studies. ${ }^{[6,10-12]}$ These symptoms were significant enough to prevent the patients' return to premorbid lifestyle. Deficits on neuropsychological testing were found in all patients. The most frequently seen deficits were verbal learning, verbal memory, sustained attention, planning and response inhibition, and the most frequent neuropsychological localization was prefrontal and left medial temporal which is similar to other reports..$^{[9,12,13]}$

Of the 59 children studied by Koelfen et al. one year after head injury, MRI lesions were seen in $66 \%{ }^{[14]}$ The distribution of the lesions was: cortical lesions in $14 \%$; sub-cortical lesions in $12 \%$, deep white matter lesions in $31 \%$, and corpus callosum lesions in 26 . van der Naalt et al., studied MRI in 67 patients within one to three months and again within six to 12 months after injury, the lesion location was predominantly in the frontal and temporal regions. ${ }^{[5]}$ In another study by Levin et al., the MRI lesion location was also in the frontal and temporal lobes. ${ }^{[15]}$ In our study in adults the lesion location was subcortical in 55\% and callosal in 5\%. Newer imaging sequences may help to maximize the sensitivity of MRI. ${ }^{[6,12,16]}$

In the series studied by Koelfen et al., patients with cortical lesions had mainly motor deficits and patients with deep white matter lesions had both motor and cognitive deficit. ${ }^{[14]}$ Hughes et al., found imaging abnormalities in $32.5 \%$ of patients and a correlation between the MRI lesions and neuropsychological deficits to frontal lobe. ${ }^{[9]}$ Frontal lesions seen in MRI done in the early period after head injury were found to be predictive of outcome in the study by van der Naalt et al., ${ }^{[5]}$ The same study also showed fronto-temporal focal atrophic lesions seen in MRI done in the later period after head injury were also predictive of outcome. Wallesch et al., noted that even in patients with clinically mild head injury, if computed tomography showed evidence of either diffuse axonal injury or focal contusions, patients were likely to have frontal dysfunction on neuropsychological tests and suggested that MRI may further improve the predictions. ${ }^{[13]}$ In a study by Wilson et al., deeper abnormalities seen in MRI 5-18 months after closed head injury, were associated with poorer performance on neuropsychological tests. ${ }^{[17]}$ Koelfen et al., reported that children with multiple lesions on MRI one year after brain injury had neuropsychological test results (all the domains) 1 to 2 standard deviations below the control group. ${ }^{[14]}$ Hoffmann et al., found MRI abnormalities in $57 \%$ but identified only a weak correlation between neuroimaging findings and neurocognitive outcome. ${ }^{[18]}$ The present study corroborates the above findings to a limited extent because, though patients with structural lesions shown on MRI showed a trend towards slower 
mental speed and a greater likelihood for developing disturbances of sleep, memory and behavior, this correlation was not statistically significant, may be due to the small cohort size.

Studies using functional MRI (fMRI) and positron emission tomography (PET) have demonstrated functional abnormalities in patients with PCS. Chen et al., have reported lower activation on fMRI scans in the dorsal and ventral lateral prefrontal regions during working memory tasks in patients with PCS. ${ }^{[19]}$ PET based measurements of regional cerebral blood flow in the right prefrontal cortex of patients with PCS during spatial working memory tasks showed a smaller increase compared to controls. ${ }^{[20]}$

One of the limitations of the present study is the absence of a control group of patients with history of MTBI but without PCS. It is possible a proportion of the lesions on MRI and neuropsychological deficits documented in this study may represent incidental findings unrelated to PCS. Only a prospective study comparing large groups of MTBI patients with and without PCS will help clarify the relative role of functional deficits and structural damage in the pathogenesis of PCS.

\section{References}

1. Kurtzke JF. The epidemiology of neurologic disease. In: RJ J, editor. Clinical neurology, rev. Philadelphia: JB Lippincott; 1993.

2. Levin HS, Mattis S, Ruff RM, Eisenberg HM, Marshall LF, Tabaddor K, et al. Neurobehavioral outcome following minor head injury: A three-center study. J Neurosurg 1987;66:234-43.

3. Ponsford J, Willmott C, Rothwell A, Cameron P, Kelly AM, Nelms R, et al. Factors influencing outcome following mild traumatic brain injury in adults. J Int Neuropsychol Soc 2000;6:568-79.

4. King N. Emotional, neuropsychological, and organic factors: Their use in the prediction of persisting postconcussion symptoms after moderate and mild head injuries. J Neurol Neurosurg Psychiatry 1996;61:75-81.

5. Van der Naalt J, Hew JM, van Zomeren AH, Sluiter WJ, Minderhoud JM. Computed tomography and magnetic resonance imaging in mild to moderate head injury: Early and late imaging related to outcome. Ann Neurol 1999;46:70-8.

6. Voller B, Benke T, Benedetto K, Schnider P, Auff E, Aichner F. Neuropsychological, MRI and EEG findings after very mild traumatic brain injury. Brain Inj 1999;13:821-7.

7. Jennett B, Bond M. Assessment of outcome after severe brain damage. Lancet 1975;1:480-4.

8. Rao SL SD, Gopukumar K. NIMHANS Neuropsychology Battery2004-Manual. $1^{\text {st }}$ ed. Bangalore: National Institute of Mental Health and Neurosciences; 2004.

9. Hughes DG, Jackson A, Mason DL, Berry E, Hollis S, Yates DW. Abnormalities on magnetic resonance imaging seen acutely following mild traumatic brain injury: Correlation with neuropsychological tests and delayed recovery. Neuroradiology 2004;46:550-8.

10. Levin HS, Gary HE Jr., Eisenberg HM, Ruff RM, Barth JT, Kreutzer J, et al. Neurobehavioral outcome 1 year after severe head injury. Experience of the Traumatic Coma Data Bank. J Neurosurg 1990;73:699-709.

11. Flanagan S. Physiatric management of mild traumatic brain injury. Mt Sinai J Med 1999;66:152-9.

12. Ruchinskas RA, Francis JP, Barth JT. Mild head injury in sports. Appl Neuropsychol 1997;4:43-9.

13. Wallesch CW, Curio N, Galazky I, Jost S, Synowitz H. The neuropsychology of blunt head injury in the early postacute stage: Effects of focal lesions and diffuse axonal injury. J Neurotrauma 2001;18:11-20.

14. Koelfen W, Freund M, Dinter D, Schmidt B, Koenig S, Schultze C. Long-term follow up of children with head injuries-classified as "good recovery" using the Glasgow Outcome Scale: Neurological, neuropsychological and magnetic resonance imaging results. Eur J Pediatr 1997;156:230-5.

15. Levin HS, Williams DH, Eisenberg HM, High WM Jr, Guinto FC Jr. Serial MRI and neurobehavioural findings after mild to moderate closed head injury. J Neurol Neurosurg Psychiatry 1992;55:255-62.

16. King NS. Post-concussion syndrome: Clarity amid the controversy? Br J Psychiatry 2003;183:276-8.

17. Wilson JT, Wiedmann KD, Hadley DM, Condon B, Teasdale G, Brooks DN. Early and late magnetic resonance imaging and neuropsychological outcome after head injury. J Neurol Neurosurg Psychiatry 1988;51:391-6.

18. Hofman PA, Stapert SZ, van Kroonenburgh MJ, Jolles J, de Kruijk J, Wilmink JT. MR imaging, single-photon emission CT, and neurocognitive performance after mild traumatic brain injury. AJNR Am J Neuroradiol 2001;22:441-9.

19. Chen JK, Johnston Km, Collie A, McCrory P, Ptito A. A validation of the post concussion symptom scale in assessment of complex concussion using cognitive testing and functional MRI. J Neurol Neurosurg Psychiatry 2007;78:1231-8.

20. Chen SHA, Kareken DA, Fastenau PS, Trexler LE, Hutchins GD. A study of persistent post-concussion symptoms in mild head trauma using positron emission tomography. J Neurol Neurosurg Psychiatry $2003 ; 74: 326-32$.

Accepted on 21-08-2009

Source of Support: Nil, Conflict of Interest: None declared. 